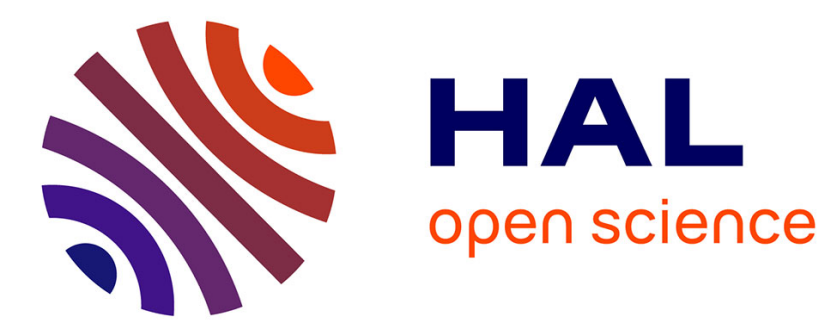

\title{
Local Exponential Stabilization of Semi-Linear Hyperbolic Systems by Means of a Boundary Feedback Control
}

Liguo Zhang, Christophe Prieur, Junfei Qiao

\section{- To cite this version:}

Liguo Zhang, Christophe Prieur, Junfei Qiao. Local Exponential Stabilization of Semi-Linear Hyperbolic Systems by Means of a Boundary Feedback Control. IEEE Control Systems Letters, 2018, 2 (1), pp.55-60. 10.1109/LCSYS.2017.2724141 . hal-01575511

\section{HAL Id: hal-01575511 \\ https://hal.science/hal-01575511}

Submitted on 21 Aug 2017

HAL is a multi-disciplinary open access archive for the deposit and dissemination of scientific research documents, whether they are published or not. The documents may come from teaching and research institutions in France or abroad, or from public or private research centers.
L'archive ouverte pluridisciplinaire HAL, est destinée au dépôt et à la diffusion de documents scientifiques de niveau recherche, publiés ou non, émanant des établissements d'enseignement et de recherche français ou étrangers, des laboratoires publics ou privés. 


\title{
Local Exponential Stabilization of Semi-Linear Hyperbolic Systems by Means of a Boundary Feedback Control
}

\author{
Liguo Zhang, Christophe Prieur, and Junfei Qiao
}

\begin{abstract}
This paper investigates the boundary feedback control for a class of semi-linear hyperbolic partial differential equations with nonlinear relaxation, which is local Lipschitz continuous with a stable matrix structure. A sufficient condition in terms of linear inequalities is developed for the existence of global Cauchy solutions and the exponential stability by seeking a balance between the relaxation term and the boundary condition. These results are illustrated with an application to the boundary feedback control for a class of hyperbolic LotkaVolterra models.
\end{abstract}

Index Terms-Semi-linear hyperbolic systems, LotkaVolterra, Lyapunov function, Boundary feedback control.

\section{INTRODUCTION}

Boundary feedback control is central for the stabilization of hyperbolic PDEs (partial differential equations). We are particularly focused on the influence of source terms on the design of boundary conditions. This problem has gained substantial interest since many engineering or physical processes may be represented by hyperbolic PDEs, such as SaintVenant equation for open channels [7], Euler equation for gas pipes [9], and Aw-Rascle equation for traffic [2].

Some significant contributions have been given in this field. A general condition using an assumption on the linear source term to be marginally diagonally stable is proposed in [6, Theorem 2]. For the linear $2 \times 2$ hyperbolic case a less restrictive sufficient condition is proposed in [3]. Strict Lyapunov functions can be defined to get input-to-state stability for time-varying linear hyperbolic PDEs with relaxation as presented in [14]. Recently, [10] derives a new stabilization result by exploiting the relaxation structure. A constructed linear Lyapunov function is presented in [17] for the positive linear hyperbolic systems. All these works investigate the boundary contractive conditions as in [4, Theorem 5.4]. However, as mentioned in [13], the source terms may be beneficial to the stability of hyperbolic PDEs and allow to construct the non-contractive boundary conditions.

In this paper, we consider a special class of semi-linear hyperbolic PDEs with nonlinear relaxation. This relaxation nonlinearity can be seen as a Lotka-Volterra type nonlinear coupling, as for instance in the models of biological networks [12], plug flow reactors [1] and Raman amplifiers [8]. The

This work is supported by the National Natural Science Foundation of China (NSFC, grant No. 61374076, No. 61533002) and the International Cooperation and Exchange Program of NSFC (grant No. 61111130119).

L. Zhang and J. Qiao are with Faculty of Information Technology, Beijing University of Technology, and with Key Laboratory of Computational Intelligence and Intelligent Systems, 100124, Beijing, China zhangliguo@bjut.edu.cn; junfeiq@bjut.edu.cn

C. Prieur is with Univ. Grenoble Alpes, CNRS, GIPSA-lab, F-38000 Grenoble, France christophe.prieuregipsa-lab.fr first contribution of this paper is the local existence of the classical solutions even if the nonlinear relaxation is only local Lipschitz continuous. We prove that the solutions of the Cauchy problem are bounded in $L^{2}$-norm. The second contribution is the linear inequality conditions for the exponential stability. More precisely, this sufficient condition depends on the balance between the relaxation term and the boundary conditions of the semi-linear hyperbolic PDEs. This is proved by designing the strictly Lyapunov function with the weighting parameters that are not restricted to be positive. Numerical computing of the inequality conditions is discussed by combining the bounded line search and the polytopic embedding techniques.

Moreover, the boundary feedback control is applied to stabilize the hyperbolic Lotka-Volterra model in which the boundary conditions are the power function type. Theoretical contributions guarantee the exponential convergence of the semi-linear hyperbolic model, even though under the nonconstructive boundary conditions.

This paper is organized as follows. The class of the semilinear hyperbolic systems with the nonlinear relaxation is given in Section II. In Section III, our main result on the sufficient condition for the exponential stability is derived. The global existence of the classical Cauchy solution also is presented. Numerical computation of the conditions is discussed in Section IV. Finally, in Section V, the boundary feedback control of the hyperbolic Lotka-Volterra model is presented.

Notation. $\mathbb{R}_{+}, \mathbb{R}^{n}$ and $\mathbb{R}^{n \times n}$ are the set of non-negative reals, $n$-order vectors and $n$-order matrices. A matrix (vector) $A$ with entries in $\mathbb{R}_{+}$is called non-negative and is denoted as $A \succeq 0$. It is said to be positive, $A \succ 0$, if all entries are positive. The expression $A \succeq B$, indicate the difference $A-B$ is non-negative. The term non-positive, negative are defined analogously as $\preceq$ and $\prec$. $\lambda_{\max }(A), \rho(A)$ stand for the largest real parts of eigenvalues and the spectral radius of $A$. Given a function $g:[0,1] \rightarrow \mathbb{R}^{n}$, we define its $L^{\infty}, L^{2}$-norm as $\|g\|_{L^{\infty}}=$ $\sup _{x \in(0,1)}|g(x)|,\|g\|_{L^{2}}=\sqrt{\int_{0}^{1}|g(x)|^{2} d x}$, respectively, where $|\cdot|$ is the Euclidean norm in $\mathbb{R}^{n}$. We call $L^{2}(0,1)$ the space of all measurable functions $g$ for which $\|g\|_{L^{2}}<\infty$.

\section{Semi-Linear Hyperbolic Systems with NONLINEAR RELAXATION}

Consider a class of semi-linear hyperbolic systems with relaxation

$$
\partial_{t} \xi+\Lambda \partial_{x} \xi=M f(\xi), \quad t \in[0, \infty), \quad x \in[0,1]
$$


where $\xi:[0,1] \times[0, \infty) \rightarrow \mathbb{R}^{n}, \Lambda$ is a diagonal matrix in $\mathbb{R}^{n \times n}$ such that $\Lambda=\operatorname{diag}\left\{\lambda_{1}, \lambda_{2}, \cdots, \lambda_{n}\right\}$, with $\lambda_{i}<0$ for $i \in\{1, \ldots, m\}$ and $\lambda_{i}>0$ for $i \in\{m+1, \ldots, n\}$. For the relaxation term, matrix $M \in \mathbb{R}^{n \times n}$ and nonlinear function $f(\xi)=\left(f_{1}\left(\xi_{1}\right), \ldots, f_{n}\left(\xi_{n}\right)\right)^{\top}$, where scalar function $f_{i}\left(\xi_{i}\right)$ is continuous to $\xi_{i}, f_{i}(0)=0$, and satisfies $\xi_{i} f_{i}\left(\xi_{i}\right)>0$, for all $\xi_{i} \neq 0$.

We use the notation $\xi=\left[\xi^{-}, \xi^{+}\right]^{\top}$ with $\xi^{-}:[0,1] \times$ $[0, \infty) \rightarrow \mathbb{R}^{m}$ and $\xi^{+}:[0,1] \times[0, \infty) \rightarrow \mathbb{R}^{n-m}$. In addition, we consider the following boundary condition

$$
\left[\begin{array}{c}
\xi^{-}(1, t) \\
\xi^{+}(0, t)
\end{array}\right]=G\left[\begin{array}{c}
\xi^{-}(0, t) \\
\xi^{+}(1, t)
\end{array}\right],
$$

where $G \in \mathbb{R}^{n \times n}$. Let us introduce the matrices $G_{--}$in $\mathbb{R}^{m \times m}, G_{-+}$in $\mathbb{R}^{m \times(n-m)}, G_{+-}$in $\mathbb{R}^{(n-m) \times m}$, and $G_{++}$in $\mathbb{R}^{(n-m) \times(n-m)}$ such that $G=\left[\begin{array}{ll}G_{--} & G_{-+} \\ G_{+-} & G_{++}\end{array}\right]$.

We shall consider the initial condition given by

$$
\xi(x, 0)=\xi^{0}(x), \quad x \in(0,1),
$$

for a given function $\xi^{0} \in L^{2}(0,1)$.

Let $|\Lambda|=\operatorname{diag}\left\{\Lambda^{-}, \Lambda^{+}\right\}$with $\Lambda^{-}=\operatorname{diag}\left\{\left|\lambda_{1}\right|, \cdots,\left|\lambda_{m}\right|\right\}$ and $\Lambda^{+}=\operatorname{diag}\left\{\lambda_{m+1}, \cdots, \lambda_{n}\right\}$, the classical definition of a solution to the Cauchy problem (1)-(3) in $L^{2}(0,1)$ is

Definition 1: Let $\xi^{0} \in L^{2}(0,1)$. A map $\xi:(0,1) \times[0, T] \rightarrow$ $\mathbb{R}^{n}$ is a solution of the Cauchy problem (1)-(3) if $\xi \in$ $C^{0}\left([0, T] ; L^{2}(0,1)\right)$ such that, for every $\varphi=\left(\varphi_{-}^{\top}, \varphi_{+}^{\top}\right)^{\top} \in$ $C^{1}\left([0,1] \times[0, T] ; \mathbb{R}^{n}\right)$ with compact support and satisfying

$$
\begin{aligned}
& {\left[\begin{array}{l}
\varphi^{-}(1, t) \\
\varphi^{+}(0, t)
\end{array}\right]} \\
& =\left[\begin{array}{cc}
\left(\Lambda^{-}\right)^{-1} G_{--}^{\top} \Lambda^{-} & \left(\Lambda^{-}\right)^{-1} G_{-+}^{\top} \Lambda^{+} \\
\left(\Lambda^{+}\right)^{-1} G_{+-}^{\top} \Lambda^{-} & \left(\Lambda^{+}\right)^{-1} G_{++}^{\top_{+}} \Lambda^{+}
\end{array}\right]\left[\begin{array}{c}
\varphi^{-}(0, t) \\
\varphi^{+}(1, t)
\end{array}\right],
\end{aligned}
$$

we have

$$
\begin{gathered}
\int_{0}^{1} \varphi^{\top}(x, T) \xi(x, T) d x-\int_{0}^{1} \varphi^{\top}(x, 0) \xi^{0}(x) d x \\
=\int_{0}^{T} \int_{0}^{1}\left[\left(\varphi_{t}^{\top}+\varphi_{x}^{\top} \Lambda\right) \xi+\varphi^{\top} M f(\xi)\right] d x d t .
\end{gathered}
$$

The global Lipschitz properties of the nonlinear relaxation guarantee the well-posedness of the global solution to the Cauchy problem (1)-(3), i.e, $T=\infty$. While, if $f$ is only locally Lipschitz continuous, such as $f(\xi)=e^{\xi}$ or $f(\xi)=\xi^{p}$ with $p>1$, the solution may blow up in finite time. In this case, we have the following results (see e.g. [5] for a wellposedness result on semi-linear hyperbolic systems and [4, Appendix B] for the quasi-linear case).

Theorem 1: If the nonlinear relaxation $f$ is locally Lipschitz continuous, then there exists $\delta>0$ such that for every $\xi^{0} \in L^{2}(0,1)$, satisfying $\left\|\xi^{0}\right\|_{L^{2}} \leq \delta$, there exists $T \in$ $[0, \infty) \cup\{\infty\}$ and a unique solution $\xi$ of Cauchy problem (1)-(3) maximally defined for $t \in[0, T)$, such that

$$
\xi \in C^{0}\left([0, T) ; L^{2}(0,1)\right),
$$

with the property that either $T=\infty$ or $T<\infty$ and $\lim _{t \rightarrow T}\|\xi(t)\|_{L^{2}}=\infty$.
From Theorem 1 it follows that the Cauchy problem (1)(3) has a unique local solution. Given an initial condition $\xi^{0}$, to prove that the solution to (1)-(3) locally defined on $[0, T)$ is maximally defined on $[0, \infty)$, it is sufficient by Theorem 1 , to prove that $\sup _{t \in[0, T)}\|\xi(t)\|_{L^{2}}$ is bounded uniformly with respect to $T$. This is indeed the case, as soon as $G$ and $M$ have some stable structures, as proved in the next section.

The definition of the local exponential stability of system (1)-(3) is as follows.

Definition 2: The steady-state $\xi=0$ of system (1)-(3) is said to be exponentially stable in $L^{2}$-norm, if there exist $v>$ $0, C>0$ and $\delta>0$ such that the solution $\xi$ of the Cauchy problem (1)-(3) is defined on $[0,1] \times[0, \infty)$ and satisfies

$$
\|\xi(t)\|_{L^{2}}^{2} \leq C e^{-v t}\left\|\xi^{0}\right\|_{L^{2}}^{2},
$$

for all $t \in[0, \infty)$ and every initial condition $\xi^{0}$ satisfying $\left\|\xi^{0}\right\|_{L^{2}} \leq \delta$.

After the previous result on the local unique solution, a constructed Lyapunov function is used in the next section to study both the global existence of the Cauchy problem and the exponential stability of system (1)-(3).

\section{Global Existence AND Boundary Stability of SEMI-LINEAR HYPERBOLIC SYSTEMS}

We start this section by giving an assumption on the structure of the boundary condition matrix and relaxation. Denote the weighting matrix $I_{n}(x)=\operatorname{diag}\left\{e^{2 \mu x} I_{m}, e^{2 \mu(1-x)} I_{n-m}\right\}$, $\mu \in \mathbb{R}$.

Assumption 1: We assume in system (1)-(3), $M:=\left(m_{i j}\right)$ is a Metzler matrix and $G:=\left(g_{i j}\right) \succeq 0$.

Theorem 2: Suppose the system (1) fulfills Assumption 1. Let $\theta=\left(\theta_{1}, \ldots, \theta_{n}\right)^{\top}, b=\left(b_{1}, \ldots, b_{n}\right)^{\top}$ be positive vectors, i.e. $\theta \succ 0, b \succ 0, \mu \in \mathbb{R}$ be a constant such that, for all $x \in(0,1)$,

$$
\begin{aligned}
\left(G^{\top}-e^{-\mu} I_{n}\right) \theta & \preceq 0, \\
\left(G-e^{-\mu} I_{n}\right) b & \preceq 0, \\
\left(|\Lambda|^{-1} M-2 \mu I_{n}\right) \theta & \prec 0, \\
M^{\top}|\Lambda|^{-1} I_{n}(x) b & \preceq 0,
\end{aligned}
$$

then the following properties hold:

- [Well-posedness] There exists $\delta>0$ such that, for every $\xi^{0} \in L^{2}(0,1)$ satisfying $\left\|\xi^{0}\right\|_{L^{2}} \leq \delta$, there exists a unique solution $\xi$ of Cauchy problem (1)-(3) defined for $t \in[0, \infty)$, such that

$$
\xi \in C^{0}\left([0, \infty) ; L^{2}(0,1)\right) .
$$

- [Exponential stability] The steady-state $\xi=0$ is locally exponentially stable for system (1)-(3), and moreover a (local) Lyapunov function is given by

$$
V=\int_{0}^{1} \xi^{\top}|\Lambda|^{-1} P I_{n}(x) \xi d x,
$$

with

$$
P=\operatorname{diag}\left\{\frac{b_{1}}{\theta_{1}}, \frac{b_{2}}{\theta_{2}}, \ldots, \frac{b_{n}}{\theta_{n}}\right\} .
$$


Proof: Let us remark that $V$ of (12) is a continuous function of $t$ by (5). In order to prove Theorem 2, pick $\delta>0$ given by Theorem 1, such that a solution to (1)-(3) exists as soon as the $L^{2}$-norm of the initial condition is smaller than $\delta$.

Part 1: Time-derivative of the Lyapunov function.

Consider a solution $\xi$ of class $C^{1}$ on $[0,1] \times[0, T]$. Under this assumption (that will be relaxed later on) $V$ is of class $C^{1}$ in $[0, T]$ and the first step of the proof is to compute the following estimation of the time-derivative $\dot{V}$ along the solution $\xi$. It yields the following:

$$
\begin{aligned}
\dot{V}= & \int_{0}^{1}\left[\xi^{\top}|\Lambda|^{-1} P I_{n}(x) \partial_{t} \xi+\partial_{t} \xi^{\top}|\Lambda|^{-1} P I_{n}(x) \xi\right] d x \\
= & \int_{0}^{1}\left[-\xi^{\top} I_{\lambda} P I_{n}(x) \partial_{x} \xi-\partial_{x} \xi^{\top} I_{n}(x) P I_{\lambda} \xi\right. \\
& \left.+f^{\top}(\xi) M^{\top}|\Lambda|^{-1} P I_{n}(x) \xi+\xi^{\top}|\Lambda|^{-1} P I_{n}(x) M f(\xi)\right] d x
\end{aligned}
$$

with $I_{\lambda}=\operatorname{diag}\left\{-I_{m}, I_{n-m}\right\}$. Then, integrating by parts, and because $\partial_{x} I_{\lambda} P I_{n}(x)=-2 \mu P I_{n}(x)$, we obtain

$$
\begin{aligned}
\dot{V}= & -\left[\xi^{\top} I_{\lambda} P I_{n}(x) \xi\right]_{0}^{1} \\
& -2 \mu \int_{0}^{1} \xi^{\top} P I_{n}(x) \xi d x+2 \int_{0}^{1} \xi^{\top}|\Lambda|^{-1} P I_{n}(x) M f(\xi) d x \\
= & \xi^{\top}(0, t) I_{\lambda} P I_{n}(0) \xi(0, t)-\xi^{\top}(1, t) I_{\lambda} P I_{n}(1) \xi(1, t) \\
& -2 \mu \int_{0}^{1} \xi^{\top} P I_{n}(x) \xi d x+2 \int_{0}^{1} \xi^{\top}|\Lambda|^{-1} P I_{n}(x) M f(\xi) d x \\
= & \dot{V}_{1}+\dot{V}_{2}+\dot{V}_{3}
\end{aligned}
$$

with

$$
\begin{aligned}
& \dot{V}_{1} \triangleq \xi^{\top}(0, t) I_{\lambda} P I_{n}(0) \xi(0, t)-\xi^{\top}(1, t) I_{\lambda} P I_{n}(1) \xi(1, t), \\
& \dot{V}_{2} \triangleq-2 \mu \int_{0}^{1} \xi^{\top} P I_{n}(x) \xi d x, \\
& \dot{V}_{3} \triangleq 2 \int_{0}^{1} \xi^{\top}|\Lambda|^{-1} P I_{n}(x) M f(\xi) d x .
\end{aligned}
$$

Under the boundary condition (2), we have

$$
\begin{aligned}
\dot{V}_{1}= & {\left[\begin{array}{c}
\xi^{-}(0, t) \\
\xi^{+}(1, t)
\end{array}\right]^{\top}\left(\left[\begin{array}{cc}
I_{m} & 0 \\
G_{+-} & G_{++}
\end{array}\right]^{\top} I_{\lambda} P I_{n}(0)\right.} \\
& {\left[\begin{array}{cc}
I_{m} & 0 \\
G_{+-} & G_{++}
\end{array}\right]-\left[\begin{array}{cc}
G_{--} & G_{-+} \\
0 & I_{n-m}
\end{array}\right]^{\top} I_{\lambda} P I_{n}(1) } \\
& {\left.\left[\begin{array}{cc}
G_{--} & G_{-+} \\
0 & I_{n-m}
\end{array}\right]\right)\left[\begin{array}{c}
\xi^{-}(0, t) \\
\xi^{+}(1, t)
\end{array}\right] } \\
= & {\left[\begin{array}{c}
\xi^{-}(0, t) \\
\xi^{+}(1, t)
\end{array}\right]^{\top}\left[\begin{array}{ll}
Q_{--} & Q_{-+} \\
Q_{+-} & Q_{++}
\end{array}\right]\left[\begin{array}{l}
\xi^{-}(0, t) \\
\xi^{+}(1, t)
\end{array}\right], }
\end{aligned}
$$

with $Q_{--}=e^{2 \mu}\left(G_{+-}^{\top} P^{+} G_{+-}+G_{--}^{\top} P^{-} G_{--}\right)-P^{-}, Q_{+-}=$ $e^{2 \mu}\left(G_{+-}^{\top} P^{+} G_{++}+G_{--}^{\top} P^{-} G_{-+}\right), Q_{-+}=Q_{+-}^{\top}$, and $Q_{++}=$ $e^{2 \mu}\left(G_{++}^{\top} P^{+} G_{++}+G_{-+}^{\top} P^{-} G_{-+}\right)-P^{+}$. Here, $P^{\mp}$ contain the corresponding $m$ and $n-m$ diagonal entries of $P$, i.e., $P=$ $\operatorname{diag}\left\{P^{-}, P^{+}\right\}$.

Denote $Q=\left[\begin{array}{ll}Q_{--} & Q_{-+} \\ Q_{+-} & Q_{++}\end{array}\right]$, then $Q$ can be rewritten as $Q=\left(e^{\mu} G\right)^{\top} P\left(e^{\mu} G\right)-P$. Multiplying $e^{\mu}>0$ on one side of inequalities (7) and (8), we have

$$
\left(e^{\mu} G-I_{n}\right) \theta \preceq 0, \quad\left(e^{\mu} G^{\top}-I_{n}\right) b \preceq 0 .
$$

Because $e^{\mu} G$ includes non-negative entries, from the PerronFrobenius theorem ([16, Theorem 2.4, p.72]), inequalities (16) are equivalent to the existence of a positive diagonal matrix $P$ defined as (13), such that matrix $Q$ is semi-definite negative. Then, it follows that $\dot{V}_{1} \leq 0$.

Let $b(x)=I_{n}(x) b$, for all $x \in(0,1)$, using inequalities (9) and (10), we have $\sum_{i=1}^{n} m_{i j}\left|\lambda_{i}\right|^{-1} b_{i}(x) \leq 0$, for all $j=1, \ldots, n$, and $\sum_{j=1}^{n} m_{i j} \theta_{j} \leq 2 \mu\left|\lambda_{i}\right| \theta_{i}-v \theta_{i}$, for all $i=1, \ldots, n$, with a sufficient small variable $v>0$, which depends on $\mu, M$ and $\Lambda$.

Since $M$ is a Metzler matrix and $\xi_{i} f_{i}\left(\xi_{i}\right)>0$, for all $\xi_{i} \neq 0$, using Jensen inequality, we obtain the estimate

$$
\begin{aligned}
\dot{V}_{3}= & 2 \int_{0}^{1} \sum_{i, j=1}^{n} \frac{b_{i}(x)}{\left|\lambda_{i}\right|} \theta_{j} m_{i j} \frac{\xi_{i}}{\theta_{i}} \frac{f_{j}\left(\xi_{j}\right)}{\theta_{j}} d x \\
\leq & \int_{0}^{1} \sum_{i=1}^{n} \frac{b_{i}(x)}{\left|\lambda_{i}\right|}\left(\frac{\xi_{i}}{\theta_{i}}\right)^{2} \sum_{j=1}^{n} m_{i j} \theta_{j} d x \\
& +\int_{0}^{1} \sum_{j=1}^{n} \theta_{j}\left(\frac{f_{j}\left(\xi_{j}\right)}{\theta_{j}}\right)^{2} \sum_{i=1}^{n} m_{i j} \frac{b_{i}(x)}{\left|\lambda_{i}\right|} d x \\
\leq & \int_{0}^{1} \sum_{i=1}^{n} \frac{b_{i}(x)}{\left|\lambda_{i}\right|}\left(\frac{\xi_{i}}{\theta_{i}}\right)^{2}\left(2 \mu\left|\lambda_{i}\right| \theta_{i}-v \theta_{i}\right) d x \\
\leq & 2 \mu \int_{0}^{1} \sum_{i=1}^{n} \frac{b_{i}(x)}{\theta_{i}} \xi_{i}^{2} d x-v \int_{0}^{1} \sum_{i=1}^{n} \frac{b_{i}(x)}{\theta_{i}}\left|\lambda_{i}\right|^{-1} \xi_{i}^{2} d x \\
\leq & -\dot{V}_{2}-v V .
\end{aligned}
$$

Finally, it follows from (14), (15) and (17) that the timederivative of $V$ satisfies

$$
\dot{V} \leq-v V .
$$

On the other hand, by remarking the definition of $V$, there exist $\alpha>0, \beta>0$ (depending on $P, \Lambda$, and $\mu$ ) such that

$$
\alpha\|\xi\|_{L^{2}}^{2} \leq V(\xi) \leq \beta\|\xi\|_{L^{2}}^{2} .
$$

The remaining part of the proof of Theorem 2 is split into two parts: 1) the Cauchy problem has a global solution, 2) the system is locally exponentially stable.

Part 2: Well-posedness proof.

Estimates (18) and (19) are obtained under the assumption that $\xi$ is of class $C^{1}$ on $[0,1] \times[0, T]$. But the selection of $\alpha$, $\beta$ and $v$ does not depend on the $C^{1}$-norm of $\xi$ : they depend only on the $C^{0}\left([0, T] ; L^{2}(0,1)\right)$-norm of $\xi$. Hence, using a classical density argument (see e.g., [4, Section 2.1.3]), the estimates (18) and (19) remain valid in the distribution sense if $\xi$ is only of class $C^{0}$.

Let $\xi \in C^{0}\left([0, T) ; L^{2}(0,1)\right)$ be the maximal solution of the Cauchy problem (1)-(3). Using estimates (18) and (19) for all $t \in[0, T)$, we get that

$$
\|\xi(\cdot, t)\|_{L^{2}}^{2} \leq \alpha^{-1} \beta e^{-v t}\left\|\xi^{0}\right\|_{L^{2}}^{2},
$$

as soon as the $L^{2}$-norm of $\xi^{0}$ is less than $\delta$. Then, using (20) and Theorem 1, we have that $T=\infty$.

Part 3: Exponential stability proof.

Consequently, if the initial condition $\xi^{0}$ has a $L^{2}$-norm smaller than $\delta$, then (20) guarantees the global solution $\xi$ 
to (1)-(3) stays in a neighborhood (with $C^{0}\left([0, \infty) ; L^{2}(0,1)\right)$ norm), while exponentially converging in $L^{2}$-norm to the origin.

This completes the proof of Theorem 2.

Remark 1: Variable $\mu$ of inequalities (7)-(9) might be positive or negative, based on the boundary condition matrix $G$ is contractive or not, which also explores the central idea of seeking a balance between the boundary condition and the relaxation. This is essential for the stability of the semi-linear hyperbolic systems.

Without a priori Assumption 1, let us deduce a corollary from the previous result.

To do that, define the auxiliary source matrix $\bar{M}=\left(\bar{m}_{i l}\right)$ with $\bar{m}_{i i}=m_{i i}$ and $\bar{m}_{i j}=\left|m_{i j}\right|$, for $j \neq i$, and boundary condition matrix $\bar{G}=\left(\bar{g}_{i j}\right)$ with $\bar{g}_{i j}=\left|g_{i j}\right|$ for all $i, j=$ $1, \ldots, n$, respectively. Thus, $\bar{M}$ is a Metzler matrix and $\bar{G}$ is a non-negative matrix.

Corollary 1: If there exist positive vectors $\theta \succ 0, b \succ$ 0 , and a constant $\mu \in \mathbb{R}$, such that the following linear inequalities hold:

$$
\begin{aligned}
\left(\bar{G}^{\top}-e^{-\mu} I_{n}\right) \theta & \preceq 0, \\
\left(\bar{G}-e^{-\mu} I_{n}\right) b & \preceq 0, \\
\left(|\Lambda|^{-1} \bar{M}-2 \mu I_{n}\right) \theta & \prec 0, \\
\bar{M}^{\top}|\Lambda|^{-1} I_{n}(x) b & \preceq 0,
\end{aligned}
$$

for all $x \in(0,1)$, then the global Cauchy solutions of (1)-(3) exist and the steady-state $\xi=0$ is exponentially stable for system (1)-(3).

Proof: Here, we also consider the Lyapunov function $V$ of (12), besides the time-derivative (14) still holds. Since the symmetric matrix $\left(e^{\mu} G\right)^{\top} P\left(e^{\mu} G\right) \leq\left(e^{\mu} \bar{G}\right)^{\top} P\left(e^{\mu} \bar{G}\right) \leq P$ holds, we obtain $\dot{V}_{1} \leq 0$.

On the other hand, linear inequalities (23) and (24) mean that $\sum_{i=1}^{n} m_{i j}\left|\lambda_{i}\right|^{-1} b_{i}(x) \leq \sum_{i=1}^{n} \bar{m}_{i j}\left|\lambda_{i}\right|^{-1} b_{i}(x) \leq 0$, and $\sum_{j=1}^{n} m_{i j} \theta_{j} \leq \sum_{j=1}^{n} \bar{m}_{i j} \theta_{j} \leq 2 \mu\left|\lambda_{i}\right| \theta_{i}-v \theta_{i}$ are satisfied, in which $v>0$ is chosen depending on $\mu, \bar{M}$ and $\Lambda$.

Therefore, the conditions (7)-(10) for system (1)-(3) are deduced from (21)-(24).

This concludes the proof of Corollary 1 .

\section{Computational Aspects}

Since inequality (10) of Theorem 2 involves the spatial variable, the number of inequality constraints is infinite. In this section, we develop some numerically computational conditions by integrating the bounded line search with the polytopic embedding.

Four vertical diagonal matrices $E_{i}, i=1,2,3,4$, are defined as $E_{1}=I_{n}, E_{2}=e^{2 \mu} I_{n}, E_{3}=\operatorname{diag}\left\{I_{m}, e^{2 \mu} I_{n-m}\right\}, E_{4}=$ $\operatorname{diag}\left\{e^{2 \mu} I_{m}, I_{n-m}\right\}$, respectively.

Proposition 1: If there exists positive vector $b \succ 0$ such that $M^{\top}|\Lambda|^{-1} E_{i} b \preceq 0$ holds for all $i=1,2,3,4$, then inequality (10) is satisfied for all $x \in(0,1)$.

Proof: For all $x \in[0,1], I_{n}(x)$ lies in the convex hull formed by the vertical matrices $E_{i}, i=1,2,3,4$. Then matrix $M^{\top}|\Lambda|^{-1} I_{n}(x), x \in(0,1)$, is embedded in the polytope formed with four vertical matrices $M^{\top}|\Lambda|^{-1} E_{i}, i=1,2,3,4$. Thus, the inequality condition (10) of Theorem 2 is satisfied as $M^{\top}|\Lambda|^{-1} E_{i} b \preceq 0$, for all $i=1,2,3,4$.

This concludes the proof of Proposition 1.

We define

$$
\begin{aligned}
& \mu_{m}=\frac{1}{2} \lambda_{\max }\left(|\Lambda|^{-1} M\right), \\
& \mu_{g}=\min _{i=1, \ldots, n}\left\{-\ln g_{i i}\right\} .
\end{aligned}
$$

Proposition 2: Let $\mu \in \mathbb{R}$, inequalities (7)-(10) of Theorem 2 are all satisfied, only if $\mu_{m}<\mu \leq \mu_{g}$.

Proof: Since $|\Lambda|^{-1} M-2 \mu I_{n}$ also is a Metzler matrix and there exists a positive vector $\theta$ such that the linear inequality (9) holds, then matrix $|\Lambda|^{-1} M-2 \mu I_{n}$ is Hurwitz. Hence, the inequality $\mu>\frac{1}{2} \lambda_{\max }\left(|\Lambda|^{-1} M\right)$ is necessary to satisfy the condition (9) of Theorem 2 .

On the other hand, since matrix $G \succeq 0$ and vector $b \succ 0$, inequality condition (7) holds only if $\mu \leq-\ln g_{i i}$, for all $i=1, \ldots, n$. Thus, $\mu \leq \min _{i=1, \ldots, n}\left\{-\ln g_{i i}\right\}$.

This concludes the proof of Proposition 2.

Proposition 3: If there exist $\mu \in\left(\mu_{m}, \mu_{g}\right]$, positive vectors $b \succ 0$ and $\theta \succ 0$, such that the conditions

$$
\begin{aligned}
\left(G^{\top}-e^{-\mu} I_{n}\right) \theta & \preceq 0, \\
\left(G-e^{-\mu} I_{n}\right) b & \preceq 0, \\
\left(|\Lambda|^{-1} M-2 \mu I_{n}\right) \theta & \prec 0, \\
M^{\top}|\Lambda|^{-1} E_{i} b & \preceq 0,
\end{aligned}
$$

hold, for all $i=1,2,3,4$, then inequalities (7)-(10) of Theorem 2 are satisfied for all $x \in(0,1)$.

Proof: The proof directly follows the results of Propositions 1 and 2 .

Remark 2: Based on the polytopic embedding method, Proposition 3 provides a numerical algorithm for solving the infinite-dimensional linear inequalities (7)-(10) by combining the bounded line search, such as Euler method, with the linear programming method.

Example 1: Let us consider the matrices

$$
\begin{gathered}
\Lambda=\operatorname{diag}\{-1,1\}, \\
M=\left[\begin{array}{rr}
-0.3 & 0.1 \\
0.1 & -0.3
\end{array}\right], \\
G=\left[\begin{array}{ll}
0.1 & 0.8 \\
0.6 & 0.4
\end{array}\right] .
\end{gathered}
$$

In this example inequality (10) is equal to the existence of $b \succ 0$ such that $\frac{1}{3} e^{4 \mu x} b_{1} \leq e^{2 \mu} b_{2} \leq 3 e^{4 \mu x} b_{1}$ holds, for all $x \in(0,1)$, in which $\mu \in(-0.1,0.9163]$ is given from (25) and (26). Let $x=0,1$ in the upper and lower bound, respectively, to form an overlap, we can easily get a feasible solution $b=(1.0011,1.0667) \succ 0$, such that condition (30) holds. *

\section{Application to Hyperbolic Lotka-Volterra MoDELS}

Consider a class of hyperbolic cooperative Lotka-Volterra model

$$
\partial_{t} w_{i}+\lambda_{i} \partial_{x} w_{i}=w_{i}\left[c_{i}+\sum_{j=1}^{n} m_{i j} w_{j}\right], \quad i=1, \ldots, n,
$$


where $w_{i}(x, t)$ is the population size of the $i$-th species, $x \in$ $[0,1], t \geq 0$. The nonlinear relaxation of system (31) is of the cooperative Lotka-Volterra type, in which $c_{i}$ is the intrinsic growth rate of each species, and $m_{i j}$ is interspecific $\left(m_{i j} \geq 0\right.$ as $i \neq j)$ or intraspecific $\left(m_{i j} \leq 0\right.$ as $\left.i=j\right)$ interaction. We assume the characteristic speeds $\lambda_{i}<0$, for $i=1, \ldots, m$, and $\lambda_{i}>0$, for $i=m+1, \ldots, n$.

For the hyperbolic system (31), there exist global positive solutions, i.e., $w=\left(w_{1}, w_{2}, \ldots, w_{n}\right)^{\top} \succ 0$, as the initial conditions are positive, $w^{0}(x) \succ 0$. We further assume that there exists a positive steady-state $w^{*} \succ 0$ for system (31), such that $c_{i}+\sum_{j=1}^{n} m_{i j} w_{j}^{*}=0$ holds, for all $i=1, \ldots, n$.

Denote $y_{i}(x, t)=\ln w_{i}(x, t)$, then (31) can be rewritten as

$$
\partial_{t} y_{i}+\lambda_{i} \partial_{x} y_{i}=c_{i}+\sum_{j=1}^{n} m_{i j} \exp \left(y_{j}(t)\right)
$$

Furthermore, let $\xi_{i}=y_{i}-y_{i}^{*}, y_{i}^{*}=\ln w_{i}^{*}$, one can obtain the hyperbolic model

$$
\partial_{t} \xi_{i}+\lambda_{i} \partial_{x} \xi_{i}=\sum_{j=1}^{n} m_{i j} f_{j}\left(\xi_{j}\right)
$$

for all $i=1, \ldots, n$, where $f_{i}: \xi_{i} \mapsto \exp \left(\xi_{i}+\ln w_{i}^{*}\right)-w_{i}^{*}$.

Then we are going to show how the boundary condition might be applied to stabilize the hyperbolic Lotka-Volterra model (31). The candidate boundary feedback strategies are considered as the power function type depending on the sign of the characteristic speeds.

For $i=1, \ldots, m$,

$$
w_{i}(1, t)=w_{i}^{*} \prod_{j=1}^{m}\left(\frac{w_{j}(0, t)}{w_{j}^{*}}\right)^{g_{i j}} \prod_{j=m+1}^{n}\left(\frac{w_{j}(1, t)}{w_{j}^{*}}\right)^{g_{i j}}
$$

and for $i=m+1, \ldots, n$,

$$
w_{i}(0, t)=w_{i}^{*} \prod_{j=1}^{m}\left(\frac{w_{j}(0, t)}{w_{j}^{*}}\right)^{g_{i j}} \prod_{j=m+1}^{n}\left(\frac{w_{j}(1, t)}{w_{j}^{*}}\right)^{g_{i j}},
$$

with feedback gains $g_{i j} \in \mathbb{R}$, for all $i, j=1, \ldots, n$.

Consequently, for the nominal system (33), we have the corresponding boundary condition as

$$
\left[\begin{array}{l}
\xi^{-}(1, t) \\
\xi^{+}(0, t)
\end{array}\right]=G\left[\begin{array}{l}
\xi^{-}(0, t) \\
\xi^{+}(1, t)
\end{array}\right],
$$

where matrix $G=\left(g_{i j}\right) \in \mathbb{R}^{n \times n}$.

The boundary feedback control (34)-(35) is now tested with some numerical simulations to illustrate our theory.

We consider a $2 \times 2$ model with two characteristic speeds $\lambda_{1}=-3, \lambda_{2}=5$, and the model entries as given as $c_{1}=$ $c_{2}=2, m_{11}=-4, m_{12}=1, m_{21}=3, m_{22}=-2$. In this case, the steady-state is obtained by $\left(w_{1}^{*}, w_{2}^{*}\right)=(1.2,2.8)$. Firstly, a non-dissipative condition matrix is considered as

$$
G=\left[\begin{array}{cc}
0.2 & 0.2 \\
0 & 1.02
\end{array}\right]
$$

in which $\rho(G)=1.02>1$. Using (25)-(26), the upper and lower bounds of $\mu$ are computed as $\mu_{m}=-0.1102$ and $\mu_{g}=-0.0198$. Solving conditions (27)-(30) of Proposition 3, we obtain $\mu=-0.0892<0, b=(2.6180,3.4319)^{\top}$ and $\theta=(0.8516,2.7451)^{\top}$. Then, Theorem 2 can guarantee the local exponential stability of steady-state $(1.2,2.8)$ of the hyperbolic system (31), (34), and (35).

To numerically compute the solutions of system (31), (34), and (35), let us discretize them using a two-step variant of the Lax-Wendroff method in [15]. We select the following initial deviations $w^{0}(x)$ from the steady-state $\left(w_{1}^{*}, w_{2}^{*}\right)=(1.2,2.8)$ as

$$
\left\{\begin{array}{l}
w_{1}(x, 0)=0.2 \sin (5 \pi x)+w_{1}^{*}, \\
w_{2}(x, 0)=0.2 \sin (5 \pi x)+w_{2}^{*} .
\end{array}\right.
$$

Figs. 1 and 2 show the time evolution of components $w_{1}$ and $w_{2}$, respectively. It is observed that $w_{1}, w_{2}$ both converge to their steady-state $w_{1}^{*}=1.2, w_{2}^{*}=2.8$ as time increases, as expected from Theorem 2 .

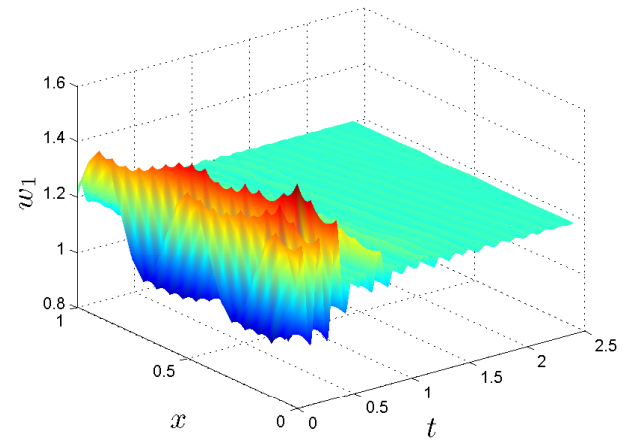

Fig. 1. The time evolution of the first component $w_{1}$ of the solution of (31), (34), and (35) with the feedback gains given by (37) and the steady-state $\left(w_{1}^{*}, w_{2}^{*}\right)=(1.2,2.8)$.

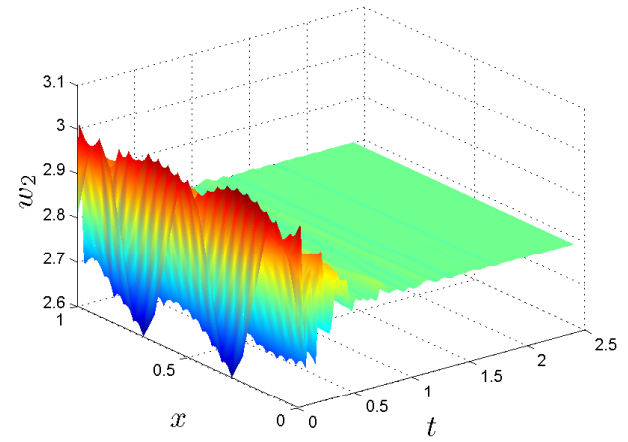

Fig. 2. The time evolution of the second component $w_{2}$ of the solution of (31), (34), and (35) with the feedback gains given by (37) and the steadystate $\left(w_{1}^{*}, w_{2}^{*}\right)=(1.2,2.8)$.

In the other simulation, we consider a hyperbolic system with a marginally stable relaxation matrix and a dissipative boundary condition matrix. In this case, the model entries are $c_{1}=-2, c_{2}=2, m_{11}=-1, m_{12}=1, m_{21}=0$, and $m_{22}=0$, then $\lambda_{\max }(M)=0$ and $\left(w_{1}^{*}, w_{2}^{*}\right)=(2,1)$ is a steady-state of system (31). Recalling (25), and since $\mu_{m}=0$, we can choose a boundary condition matrix as

$$
G=\left[\begin{array}{ll}
0.2 & 0.4 \\
0.6 & 0.2
\end{array}\right]
$$




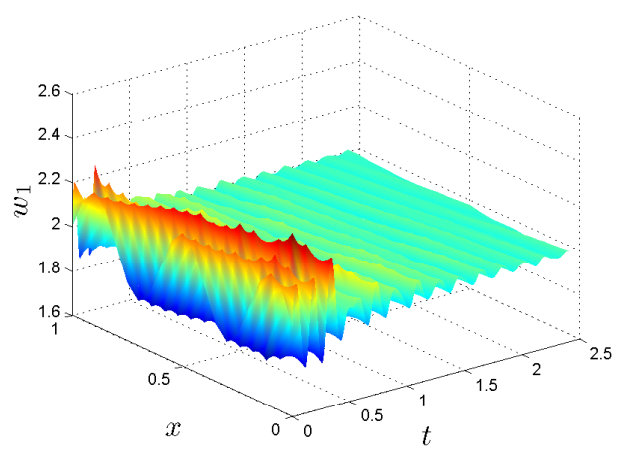

Fig. 3. The time evolution of the first component $w_{1}$ of the solution of (31), (34), and (35) with the feedback gains given by (38) and the steady-state $\left(w_{1}^{*}, w_{2}^{*}\right)=(2,1)$.

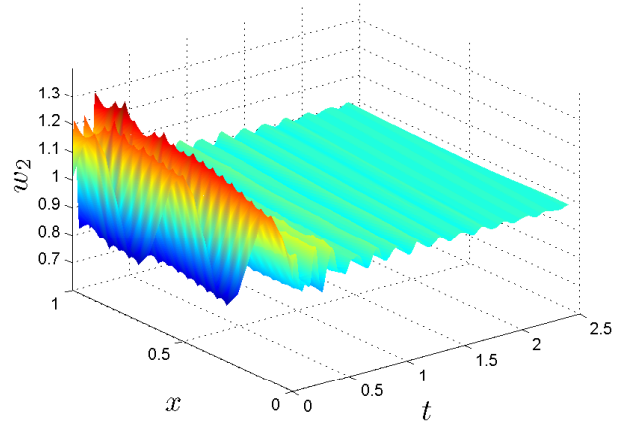

Fig. 4. The time evolution of the second component $w_{2}$ of the solution of (31), (34), and (35) with the feedback gains given by (38) and the steadystate $\left(w_{1}^{*}, w_{2}^{*}\right)=(2,1)$.

Then, calculating (26), $\mu_{g}=1.6094$. Solving (27)-(30) of Proposition 3, $\mu=0.1912>0, b=(0.6768,0.7232)^{\top}, \theta=$ $(1.2084,1.0082)^{\top}$. Hence $\left(w_{1}^{*}, w_{2}^{*}\right)=(2,1)$ is locally exponentially stable. The time evolutions of both converging components are depicted in Figs. 3 and 4, respectively.

The above two simulations show an interesting result that the nonlinear relaxation might be beneficial (as $\mu<0$ ) to the boundary feedback control of the semi-linear hyperbolic systems, by a stable matrix structure, and the strictly contractive boundary condition is not necessarily required.

\section{CONCLUSION}

This paper is concerned with a class of semi-linear hyperbolic PDEs. The global existence of the classical solution is given and the sufficient conditions are derived for the local exponential stability as the boundary condition matrix and the relaxation matrix have the stable structures. The boundary feedback control is applied to stabilize a class of hyperbolic Lotka-Volterra model.

Future work will be devoted to the study of hyperbolic balance laws with a general relaxation, in which the sumsof-square programming method in [11] can be used for the numerical computation.

\section{REFERENCES}

[1] I. Aksikas, J.J. Winkin, and D. Dochain. Optimal LQ-feedback regulation of a nonisothermal plug flow reactor model by spectral factorization. IEEE Transactions on Automatic Control, 52(7):1179$1193,2007$.

[2] A. Aw and M. Rascle. Resurrection of 'second order' models of traffic flow. SIAM Journal on Applied Mathematics, 60(3):916-938, 2000.

[3] G. Bastin and J. M. Coron. On boundary feedback stabilization of non-uniform linear $2 \times 2$ hyperbolic systems over a bounded interval. Systems \& Control Letters, 60(11):190-196, 2011.

[4] G. Bastin and J.-M. Coron. Stability and Boundary Stabilization of 1-D Hyperbolic Systems. Progress in Nonlinear Differential Equations and Their Applications. Springer, 2016.

[5] G. Bastin and J.-M. Coron. Exponential stability of semi-linear onedimensional balance laws. In N. Petit, editor, Feedback Stabilization of Controlled Dynamical Systems: In Honor of Laurent Praly, pages 265-278. Springer, 2017.

[6] A. Diagne, G. Bastin, and J.-M. Coron. Lyapunov exponential stability of 1-D linear hyperbolic systems of balance laws. Automatica, 48(1):109-114, 2012.

[7] V. Dos Santos and C. Prieur. Boundary control of open channels with numerical and experimental validations. IEEE Transactions on Control Systems Technology, 16(6):1252-1264, 2008.

[8] P. W. Dower and P. M. Farrel. On linear control of backward pumped Raman amplifiers. In IFAC Symposium on System Identification, pages 547-552, Newcastle, Australia, 2006.

[9] M. Gugat, M. Dick, and G. Leugering. Gas flow in fan-shaped networks: classical solutions and feedback stabilization. SIAM Journal on Control and Optimization, 49(5):2101-2117, 2011.

[10] M. Herty and W-A. Yong. Feedback boundary control of linear hyperbolic systems with relaxation. Automatica, 69:12-17, 2016.

[11] Jean B. Lasserre. A sum of squares approximation of nonnegative polynomials. SIAM Rev., 49(4):651C669, 2007.

[12] L. Pavel and L. Chang. Lyapunov-based boundary control for a class of hyperbolic Lotka-Volterra systems. IEEE Transactions on Automatic Control, 57(3):701-714, 2012.

[13] C. Prieur, A. Girard, and E. Witrant. Stability of switched linear hyperbolic systems by Lyapunov techniques. IEEE Transactions on Automatic control, 59(8):2196-2202, 2014.

[14] C. Prieur and F. Mazenc. ISS-Lyapunov functions for time-varying hyperbolic systems of balance laws. Mathematics of Control, Signals, and Systems, 24(1):111-134, 2012.

[15] L.F. Shampine. Solving hyperbolic PDEs in Matlab. Appl. Numer. Anal. \& Comput. Math., 2:346-358, 2005.

[16] D. D. Siljak. Decentralized Control of Complex Systems. Academic Press, New York, 1991.

[17] L. Zhang and C. Prieur. Necessary and sufficient conditions on the exponential stability of positive hyperbolic systems. IEEE Transactions on Automatic Control, 62(7):3610-3617, 2017. 